\title{
Simple homogenization-topology optimization approach for the pushover analysis of masonry walls
}

\author{
by \\ Gabriele MILANI ${ }^{(1)}$ and Matteo BRUGGI ${ }^{(2)}$ \\ (1) Corresponding author, Department ABC, Technical University of Milan, Piazza Leonardo \\ da Vinci 32, Milan, Italy. E-mail: gabriele.milani@polimi.it \\ (2) Department of Civil and Environmental Engineering DICA, Technical University of Milan, \\ Piazza Leonardo da Vinci 32, Milan, Italy
}

\begin{abstract}
A topology optimized rigid triangular FE macro-model with non-linear homogenized interfaces for the pushover analysis of in plane loaded masonry is presented. The shape of the mesh and the position of the interfaces is evaluated through a topology optimization approach that detects the main compressive stress fluxes in the structure. Different values of the horizontal action are considered to derive an adaptive mesh or an optimal discretization that is suitable for multiple loads. Masonry properties are calibrated by means of a homogenization approach in the non-linear range. To tackle elastic and inelastic deformations, interfaces are assumed to behave as elasto-plastic with softening in both tension and compression, with orthotropic behavior. The two-step procedure competes favorably with classic equivalent frame approaches because it does not require a-priori assumptions on the mesh and on the length of the rigid offsets. An example of technical relevance is discussed, relying into a multi-story masonry wall loaded up to failure.
\end{abstract}

\section{INTRODUCTION}

Unreinforced masonry is a popular construction technique for existing residential buildings in the European Alps and Mediterranean countries. For many of them where the seismicity ranges from moderate to high (such as Italy, Slovenia, Greece, Turkey), the seismic assessment of existing masonry is a task of key societal importance. The seismic performance of existing buildings is usually evaluated, in agreement with national codes, through pushover analyses, which allow approximating the ultimate lateral force distribution and displacement capacity of the building, assuming a suitable non linear material behavior, possibly with softening. The most diffused way to deal with pushover in practical design is the so called utilization of the equivalent frame approach, see for instance Addessi et al. (2015), Milani et al. (2009), Belmouden and Lestuzzi (2009), Pasticier et al. (2008), Salonikios et Al. (2003), just to cite a small sample of the existing literature. Similar approaches are based on the so-called utilization of macro-elements, see e.g. Rinaldin et al. (2016) or Chen et al. (2008), as for instance the successful TREMURI approach by Lagomarsino (Lagomarsino et al. 2013). The declared attempt is the need to simplify the complexity of the two/three dimensional model in order to be able to provide pushover curves reasonably predictive of the real behavior of the structure under increased horizontal loads, see e.g. Magenes and Della Fontana (1998) and Galasco et al. (2004). As far as the equivalent frame is concerned (but similar remarks 
could be repeated also for macro-elements), the major advantage stands in the utilization of a beam assemblage model to deal with the global behavior of a masonry wall/building, which is intrinsically two/three-dimensional, with the undoubtable simplification to lump all the inelasticity, including softening, on concentrated hinges. Such approach is quite successful, because it needs only users familiar with incremental procedures applied on frames, a skill normally achieved in undergraduate r.c. courses in engineering faculties. However, most of the scientific community does not consider the reduction of masonry walls to an assemblage of beams and rigid offsets an affordable way to describe the inelastic behavior of masonry under static loads. Also, it is well known that the reduction to an equivalent frame is not always univocal. Commercial codes sometimes do not allow the users to conceive their own frames, furthermore they require many inelastic and algorithm parameters that may turn out to provide quite different global pushover curves, see for instance Marques and Lourenço (2011) or Clementi et al. (2016). An open issue remains also the introduction of rigid offsets on all those regions being the intersection of spandrels and piers, and how such offsets may affect the global behavior. Finally, to deal with irregularities (quite common on existing buildings) is not always an easy task and several simplifications of the real geometry are needed.

On the other hand, the analysis by means of 2D/3D FEM discretization with materials exhibiting softening and orthotropic behavior (typical of masonry arranged in regular textures both in the elastic and inelastic range) may still require both prohibitive computational efforts in several cases of technical relevance (and hence powerful workstations) and a strong background on masonry modelling by the user, two conditions rarely satisfied together. For the reasons mentioned above, this latter approach still cannot be proposed in conventional design.

In order to circumvent the limitations of the equivalent frame and get all the advantages of a full 2D advanced modelling, without bringing together all the aforementioned disadvantages, here a new simple two-step procedure basing on topology optimization and homogenization is proposed for a fast and reliable 2D pushover analysis of full scale masonry walls.

The approach is indeed a two-step model which bases on a preliminary topology optimization performed on a quasi no-tension material and the subsequent discretization with rigid triangular elements and non-linear homogenized interfaces suitable for a fast in-plane pushover analysis of masonry walls.

The topology optimization (step one), see e.g. Bendsøe (1989) to Zhou \& Rozvany (1991), allows for a precise identification, under a given distribution of vertical (assumed fixed) and horizontal (a fraction of the vertical ones) loads of optimal load paths (compressed struts) dependent on the amount of lateral loads applied. This would suggest the adoption of an adaptive strategy to derive an appropriate mesh for each one of the load steps considered in the pushover analysis. Alternatively, one could use an optimization approach for multiple loads that derives a single mesh that is suitable for all the considered load cases.

Standard post-processing techniques allow deriving for each topology path contour plots of the optimal struts, whose axes can be used to generate automatically a triangular mesh with some of the element edges standing on the contour lines.

Typically, the topology optimization is repeated on the same structure at different values of the horizontal loads, providing a sequence of compressed struts configurations, which evolve and incline (to properly withstand the application of the forces) progressively increasing the amount of horizontal loads applied.

At a structural level, the analyses (step 2) are performed by means of a FE discretization made by rigid triangular elements and softening interfaces. Within a discretization made by rigid elements and interfaces where all dissipation is concentrated, the shape and position of the elements are crucial for a correct evaluation of failure mechanisms, ductility and load carrying capacity, see Milani (2011), Milani \& Tralli (2011, 2012). The idea is therefore that the position of the elements is evaluated step by step through a topology optimization, able to quickly generate compression-only load paths in the masonry structure to be analyzed, Bruggi \& Duysinx (2013). The compressed struts implicitly capture the edges and the shapes of the triangles to be used at a structural level. Having 
the aim of tackling elastic and inelastic strains, interfaces among adjoining triangles are assumed to behave elasto-plastically with softening in both tension and compression, with peak tensile strength almost zero. The mesh used smoothly evolves from one configuration to the successive, driven by topology optimization outcome in agreement with the amount of the horizontal load applied and corresponding result obtained in step 1. Such evolutionary mesh adjustment is always possible simply moving nodes of some elements from the previous to the successive configuration applying a weighed linear interpolation.

The proposed procedure competes favorably with classic equivalent frame approaches, since it does not require any a-priori assumption on the mesh or on the length of the rigid offsets. In fact, cross regions do not exist in the implemented approach and the procedure is native twodimensional. Furthermore, excellent stability of the algorithm is experienced. A relevant example is discussed in the sequel to benchmark the approach on a large scale wall with a variety of existing numerical results available from the literature (Milani et al. 2009), with promising comparisons with results obtained via different equivalent frame procedures.

\section{A TWO-STEP METHOD}

In the present Section, the two-step procedure adopted to analysis under increasing static loads large scale 2D masonry walls is discussed, starting from the topology optimization of quasi notension materials (step 1) and ending with the homogenized pushover analysis at a global scale (step 2). The interfacing procedure between step 1 and step 2 is also discussed and properly commented.

As detailed below, step 1 is implemented distributing a limited amount of linear elastic material whose negligible strength in tension is enforced through a suitable set of stress constraints. This approach, based on linear elasticity, is particularly efficient in deriving equilibrated strut-only structures. These minimum energy no-tension solutions can be seen as optimal load paths for walls whose strength in tension can be neglected, see also Bruggi (2016). This prevailing source of nonlinearity is therefore fully taken into account in this step. Step 2 consists of a more accurate formulation that handles elasto-plastic interfaces with softening in both tension and compression, with peak tensile strength almost zero. This provides a suitable insight in the non-linear behavior of the structure, especially when investigating the wall at incipient collapse.

\subsection{Step 1: The topology optimization problem}

Topology optimization is an established mathematical tool that allows distributing a prescribed amount of isotropic material such that the so-called structural compliance is minimized, i.e. the work of the external forces at equilibrium, see the original formulation by Bendsøe (1989).

Truss-like structures arise as a result of the optimization as the stiffest way to carry the load. Minimizing the structural compliance simply means minimizing the overall elastic strain energy.

Indeed, topology optimization can be adopted to select minimum energy load paths that can be seen as optimal Strut-and-Tie Models (STMs), see among the others Liang et al. (2000) and Kwak \& Noh (2006).

$$
\left\{\begin{array}{c}
\min _{x_{\min }} \leq x_{e} \leq 1 \\
\text { s.t. } \sum_{e}^{N} x_{e}^{p} \boldsymbol{K}_{0 e} \mathbf{U}_{e}=\mathbf{F} \\
W / W_{0} \leq V_{f}
\end{array}\right.
$$


Technical codes suggest the adoption of any optimization strategy that implements the elastic strain energy as objective function to deploy optimal STMs. This formulation is herein recalled for completeness sake. A minimization problem is formulated that reads:

In Eqn. (1), the objective function is the structural compliance $C=\mathbf{F}^{T} \mathbf{U}_{e}$, whereas Eqn. (1.2) enforces the discrete equilibrium within the usual displacement-based framework. Four-node finite elements are used in the simulations presented next. The stiffness matrix computed for the virgin material $\boldsymbol{K}_{0 e}$ is penalized by means of the term $x_{e}^{p}$ (with $p=3$ ), according to the Solid Isotropic Material with Penalization (SIMP) see e.g. Zhou \& Rozvany (1991). A lower bound $x_{\min } \geq 0$ is needed to avoid singularity of the discrete problem, being $x_{e}$ the minimization unknown, i.e. the density of the material in the $e$-th finite element. Eqn. (1.3) enforces the volume constraint, which is a percentage of the whole amount of material. The weight $W$ is computed multiplying the element density $x_{e}$ for the volume $V_{e}$ over the $N$ elements of the mesh, whereas $W_{0}$ denotes the volume of the design domain.

Eqn. (1) distributes a limited amount of material in order to minimize the overall strain energy of the design domain. This provides equilibrated truss-like structures that can be seen as optimal load paths.

The conventional formulation presented above does not account for any strength requirement addressing the collapse of the structure. Also, it can handle only one load case. Failure constraints should be added to tackle the former issue, whereas an extension to multiple load cases should be provided to overcome the latter.

An ad hoc problem, i.e. a suitable minimum compliance formulation with weight and stress constraints, is herein formulated to solve both points. It reads:

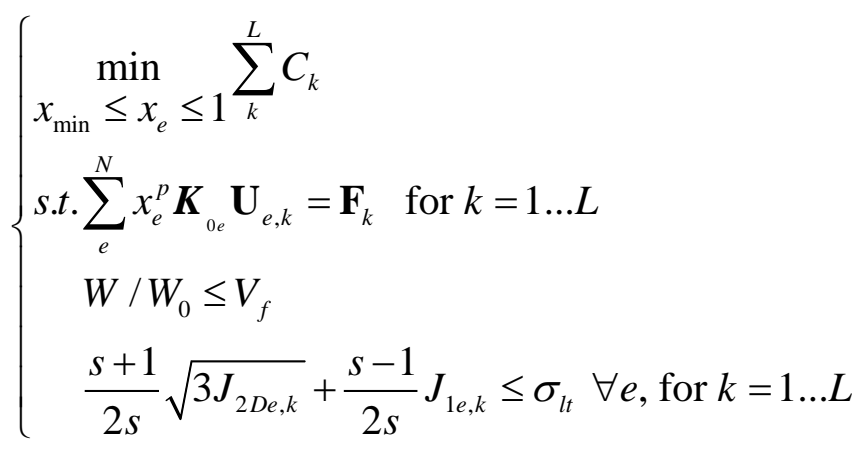

Eqn. (2) is especially conceived to cope with $L \geq 1$ load cases. For the $k$-th load case, the load vector $\mathbf{F}_{k}$ is assembled and the equilibrium can be solved to compute the relevant element displacements $\mathbf{U}_{e, k}$, see Eqn. (2.2).

The objective function is computed as the sum over the $L$ load cases of the values of the compliance $C_{k}=\mathbf{F}_{k}{ }^{T} \mathbf{U}_{e, k}$. As before, Eqn. (2.3) enforces the volume constraint, whereas Eqns. (2.4) include a set of local stress constraints. These enforcements adopt the equivalent Drucker-Prager stress measure to account for the unequal behavior in tension and compression of the masonry structure, see in particular Bruggi \& Duysinx (2013). In Eqn. (2.4), $J_{1, k}$ is the first stress invariant computed post-processing $\mathbf{U}_{e, k}, J_{2 D, k}$ the second deviatoric invariant and $\sigma_{l t}$ the strength limit in tension. The parameter $s=\sigma_{l c} / \sigma_{l t}$ is the uniaxial asymmetry ratio of the stress limit in compression $\sigma_{l c}$ to $\sigma_{l t}$.

It can be shown that the formulation in Eqn. (2), along with a suitable setup of the involved material parameters, allows coping with the optimal design of truss-like structures made of unilateral 
material. This task is accomplished within a standard stress-based scheme. Adopting small values of the strength limit in tension $\sigma_{l t}$ in conjunction with very large values of the uniaxial asymmetry ratio, an optimal design made of struts only is expected to arise.

Hence, Eqn. (2) distributes a limited amount of no-tension material in order to minimize the overall strain energy getting rid of any tensile member. This provides equilibrated truss-like structures that can be seen as compression-only optimal load paths. Adopting $L=1$, a single load case in considered in the optimization. For $L>1$ an optimal strut-only path is achieved that is well-suited to handle each one of the considered load cases.

The multi-constrained problem arising in Eqn. (2) is solved through mathematical programming, see in particular Svanberg (1989).

It is finally remarked that a robust modeling and optimization involving no-tension materials (not only strut-only models) can be alternatively achieved, for instance, adopting suitable energybased formulations that drop the Solid Isotropic Material with Penalization (SIMP) in favor of a suitable orthotropic material model. Further details can be found in Bruggi $(2014,2016)$.

\subsection{Step 2: Pushover analyses with triangular rigid elements and interfaces}

The FE model utilized at a structural level is based on a rigid triangular discretization interacting through non-linear interfaces, where shear and normal behavior are uncoupled for the sake of simplicity. The displacement field is thus assumed, inside each element $M$, dependent on centroid inplane displacements ( $u_{x}^{M}$ and $u_{y}^{M}$, indicating horizontal and vertical displacement respectively) and rotation $\Phi_{z}^{M}$ of the element around centroid, see Figure $1 u_{x}^{M}, u_{y}^{M}$ and $\Phi_{z}^{M}$ are collected in the unknowns vector $\mathbf{U}_{M}^{T}=\left[u_{x}^{M} ; u_{y}^{M} ; \Phi_{z}^{M}\right]$ for element $M$.

Focusing on an edge $\Gamma_{12}^{e}$ of $M$ (centroid $\mathbf{G}_{M}=\left[x_{M} ; y_{M}\right]$ ), the displacement field of points belonging to the edge is expressed in global coordinates as:

$\left[\begin{array}{l}u_{x 0} \\ u_{y 0}\end{array}\right]=\left[\begin{array}{ccc}1 & 0 & -\left(y_{0}-y_{M}\right) \\ 0 & 1 & \left(x_{0}-x_{M}\right)\end{array}\right]\left[\begin{array}{c}u_{x}^{M} \\ u_{y}^{M} \\ \Phi_{z}^{M}\end{array}\right]=\mathbf{R}_{M}\left(\mathbf{P}_{0}\right) \mathbf{U}_{M}$

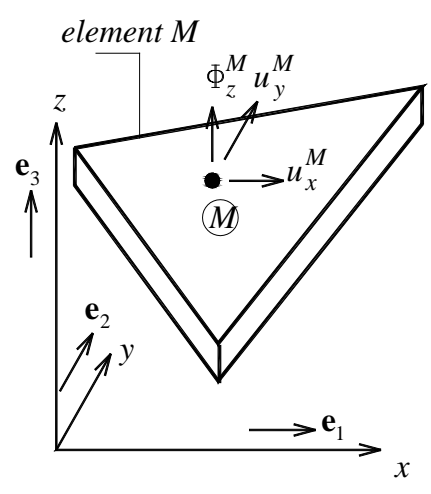

(a)

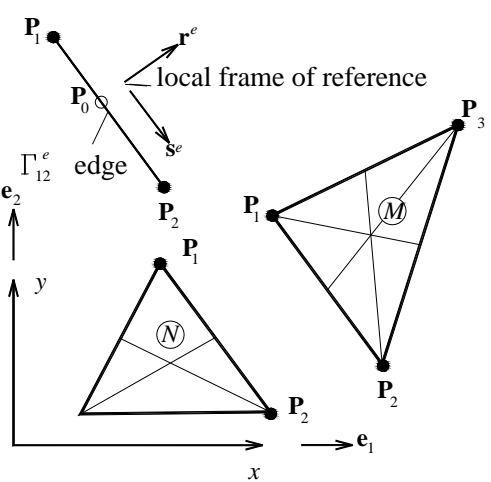

(b)

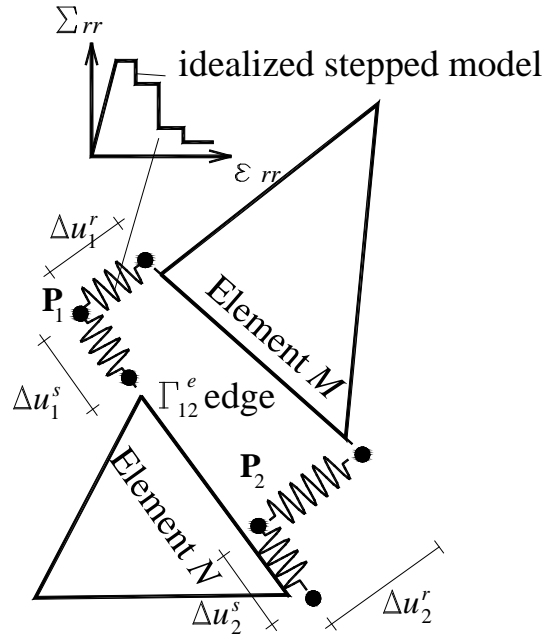

(c) 
Figure 1: Triangular rigid element used for the FE non linear analyses. -a: global optimization unknowns per element. b: local frame of reference of 1-2 edge between contiguous elements M-N. -c: jump of displacements on 1-2 edge and non-linear idealized stress-strain behavior.

Where $x_{0}$ and $y_{0}$ are global coordinates of a point $\mathbf{P}_{0}$ on $\Gamma_{12}^{e}, u_{x 0}$ and $u_{y 0}$ are the horizontal and vertical global displacement of point $\mathbf{P}_{0}$ respectively. A local frame of reference for $\Gamma_{12}^{e}$ is also needed, identified by unitary vectors $\mathbf{s}^{e}$ and $\mathbf{r}^{e}$, respectively parallel and perpendicular to the edge, with $\mathbf{s}^{e}=\left(\mathbf{P}_{2}-\mathbf{P}_{1}\right) /\left\|\mathbf{P}_{2}-\mathbf{P}_{1}\right\|$ and $\mathbf{r}^{e}=\mathbf{s}^{e} \times \mathbf{e}_{3}$. The transformation matrix from the global to the interface local coordinate system is:

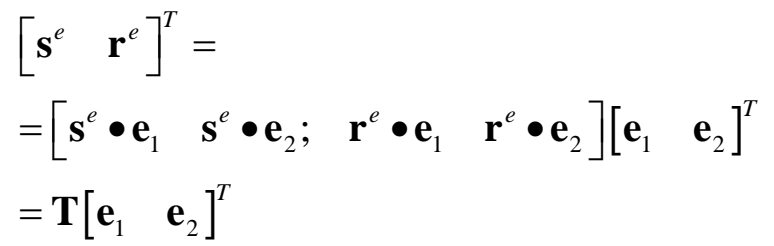

where • indicates the internal product. When two contiguous elements $M$ and $N$ with common interface $\Gamma_{12}^{e}$ are considered, the jump of displacements at the common interface can be written as:

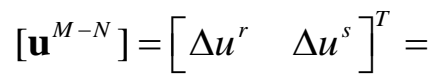

$$
\begin{aligned}
& \mathbf{T}^{-1}\left[\mathbf{R}_{M}\left(\mathbf{P}_{0}\right) \mathbf{U}_{M}-\mathbf{R}_{N}\left(\mathbf{P}_{0}\right) \mathbf{U}_{N}\right]
\end{aligned}
$$

where $\Delta u^{r}$ and $\Delta u^{s}$ are the normal and tangential jump of displacement field on the interface respectively. Due to the assumption of a rigid-infinitely resistant behavior for the triangular elements, elastic deformation and plastic dissipation occurs only along each interface $I$ between two adjacent triangles $M$ and $N$. Internal work $W_{I}^{\text {in }}$ stored in each interface $I$ can be written as follows:

$W_{I}^{\text {in }}=\int_{0}^{L_{I}}\left[N_{I}(\xi) \Delta u^{r}(\xi)+T_{I}(\xi) \Delta u^{s}(\xi)\right] d \xi$

where $N_{I}(\xi)$ and $T_{I}(\xi)$ are normal and tangential forces acting on interface $I$ at abscissa $\xi$ (varying from 0 to the length of the interface $L_{I}$ ) respectively. To solve the non-linear incremental problem, here a consolidated numerical approach applied in a variety of problems involving masonry elements and entire structures is adopted.

As demonstrated in a general framework, the incremental solution of an elasto-plastic problem may be obtained by means of the following equivalent quadratic programming problem:

$$
\left\{\begin{array}{c}
\left\{-\frac{1}{2}\left(\boldsymbol{\lambda}^{E}\right)^{T} \mathbf{H}^{E} \boldsymbol{\lambda}^{E}+\left(\boldsymbol{\lambda}^{E}\right)^{T}\left(\mathbf{N}^{E}\right)^{T} \mathbf{D}^{E} \boldsymbol{\varepsilon}^{E}\right. \\
\text { subject to }: \boldsymbol{\lambda}^{E} \geq \mathbf{0}
\end{array}\right.
$$

where $\mathbf{D}^{E}$ is the elastic stiffness matrix, $\boldsymbol{\varepsilon}^{E}$ is the elastic part of the strain vector of the element $E$, $\mathbf{N}^{E}$ is the shape function matrix of the used finite element, $\lambda^{E}$ is the plastic multiplier vector and $\mathbf{H}^{E}$ is the hardening matrix, which in this case is diagonal and with non-null very small values, with the aim of reproducing the elastic-perfectly plastic case through positive-defined matrices. Hypotheses at the base of Figure 1 and (4) are that elasto-plastic materials with associated flow rule are treated, the plasticity condition is piecewise-linearized with $r$ linearly elastic-plastic interacting 
planes, unloading of yielded stress-points does not occur and that the continuum is discretized into constant strain and stress finite elements.

Softening is dealt with in the model by means of a heuristic but very efficient numerical algorithm, as widely discussed in Milani \& Tralli (2011, 2012), where the reader is referred for further details. The core of the algorithm is a sequential quadratic programming procedure, where the uniaxial stress-strain tangential and normal constitutive relationships of the interfaces are approximated by means of linear-piecewise constant functions. In this way, at each iteration during an increment of the load, the system may be regarded as elastic-perfectly plastic. In such a condition, quadratic programming problem (4) holds, whereas the ductility limit is a-posteriori checked by means of a simple bisection procedure. Once that one of the interfaces reaches one ductility limit, the successive increment is again characterized by interfaces behaving elasto-plastically, but for all those interfaces where a ductility limit has been previously reached, a reduced value of strength is used. Refinement of the linear-piecewise approximation may be increased ad-libitum, but a good balance between real behavior and numerical efficiently is obtained using approximations not exceeding 5-10 ductility limits per interface.

It is finally remarked that the assumed rigid triangles can interpenetrate to allow deformations of the wall. Although this is questionable from a purely theoretical point of view, it can be accepted by considering that in practical applications interfaces are indeed interphases, i.e. they have a thickness that can accommodate the apparent interpenetration.

\subsection{Interface between Step 1 and Step 2: selection of the mesh and evolutionary strategy}

The interfacing procedure between step 1 and step 2 is fully automatic and handled in the present paper in a Matlab environment.

As already pointed out, the topology optimization allows for a precise identification of optimal load paths, guaranteeing the equilibrium under the applied external loads with a material unable to withstand tensile stresses. For practical purposes, this condition translates in the identification of an assemblage of compressed struts, whose position and geometry turns out to be strongly dependent on the amount of lateral loads applied. Such dependence intuitively suggests also the adoption of an adaptive strategy to derive an appropriate mesh for each one of the load steps considered in the pushover analysis.

At a structural level, the analyses (step 2) are performed by means of a FE mesh made by rigid triangular elements and softening interfaces. Within a discretization made by interfaces where all dissipation concentrates, the position of the elements is crucial for a correct evaluation of the nonlinear behavior of the structure and the compressed struts obtained in step 1 implicitly capture the edges and the shapes of the triangles to be used at a structural level.

As schematically indicated in Figure 2, standard post-processing techniques allow deriving for each topology path a series of contour plots with the optimal struts, whose axes can be used to generate automatically a triangular mesh with some edges standing on the contour lines. Automatic mesh generation is handled by means of consolidated Delaunay triangulation routines already available in Matlab.

Typically, the topology optimization is repeated on the same structure at different values of the horizontal loads, providing a sequence of compressed strut configurations, which evolve and incline (to properly withstand the application of the forces) progressively increasing the amount of horizontal loads applied.

The mesh used smoothly evolves from one configuration to the successive following the general scheme of Figure 3, driven by topology optimization outcome in agreement with the amount of the horizontal load applied and corresponding result obtained in step 1. Such evolutionary mesh adjustment is always possible simply moving nodes of some elements from the previous to the successive configuration, applying for the adaptation rule a simple weighed linear interpolation, where the weighting factor is ruled by the value of the horizontal load applied. 
It is worth noting that the procedure requires some attention, firstly because the new position of the nodes undergoing adaptation must belong to the new strut contour, but with coordinates that must be a-priori chosen by the user. Second because step by step it is necessary to re-calculate the jumps of displacement on all interfaces, being their orientation changed and being the state of stress and damage not a priori known. Again this is possible because rotations and centroid displacements of the elements are known at the previous step and hence, through Eq. ( 5 ) it is possible to estimate with a suitable post processing routine the jumps of displacement at the previous iteration, with varied position of the nodes.

Alternatively, the contour plots for all desired levels of horizontal loads that one wants to consider in the structural analyses, can be superimposed without any mesh adaptation and a single Delaunay tessellation can be performed accounting for all the different positions of the struts. However authors experienced that this last strategy may result in excessively refined discretization and irregular mesh density in all those zones where struts at different instants superimpose, needing more time to be performed and providing from practical purposes almost the same result.

It is finally remarked that the above procedure does not include an ad hoc control of the aspect ratio of the rigid elements of the mesh (ratio of longest to the shortest side in a triangle). This implies that interfaces with very different length can arise in the discretization, possibly affecting the accuracy of the achieved results. This issue can be easily solved refining the elements exhibiting high aspect ratios. The preliminary version of the algorithm used to perform the simulations presented next does not include this numerical recipe. It will be implemented to perform further investigations and provide a range of critical values of the aspect ratio that should not be overcome to preserve the required accuracy.

(a)

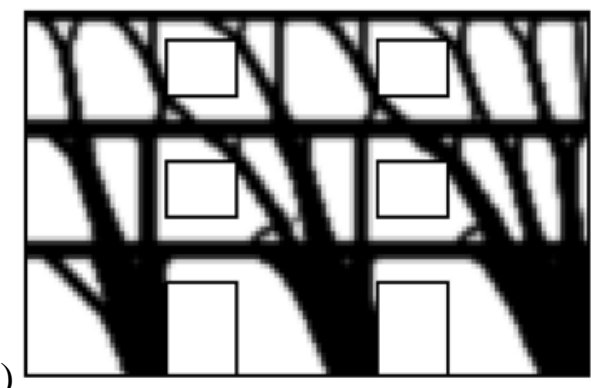

(b)

(c)

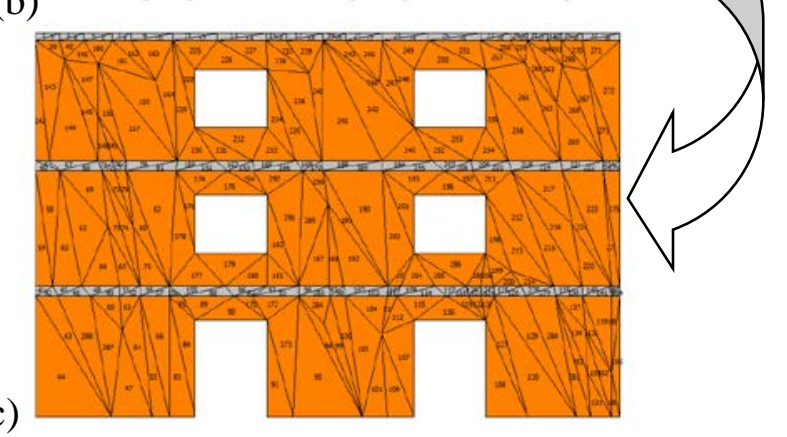

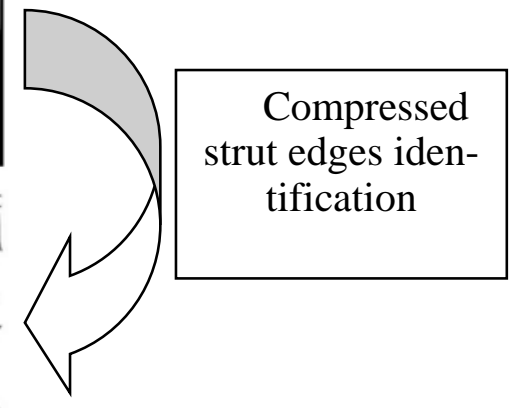

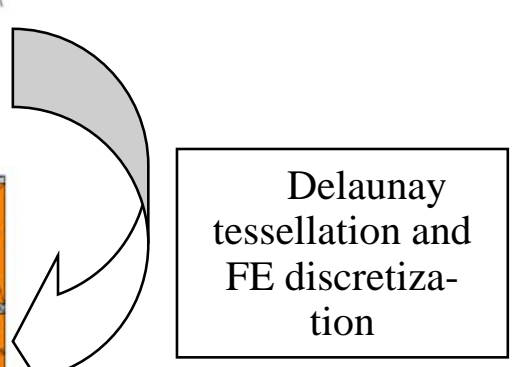


Figure 2: -a: typical result of the topology optimization for multiple loads, compression-only material model -b: edges of the optimal struts recovered from the topology optimization result $-\mathrm{c}$ : very coarse FE triangular grid derived from the optimal load path. 


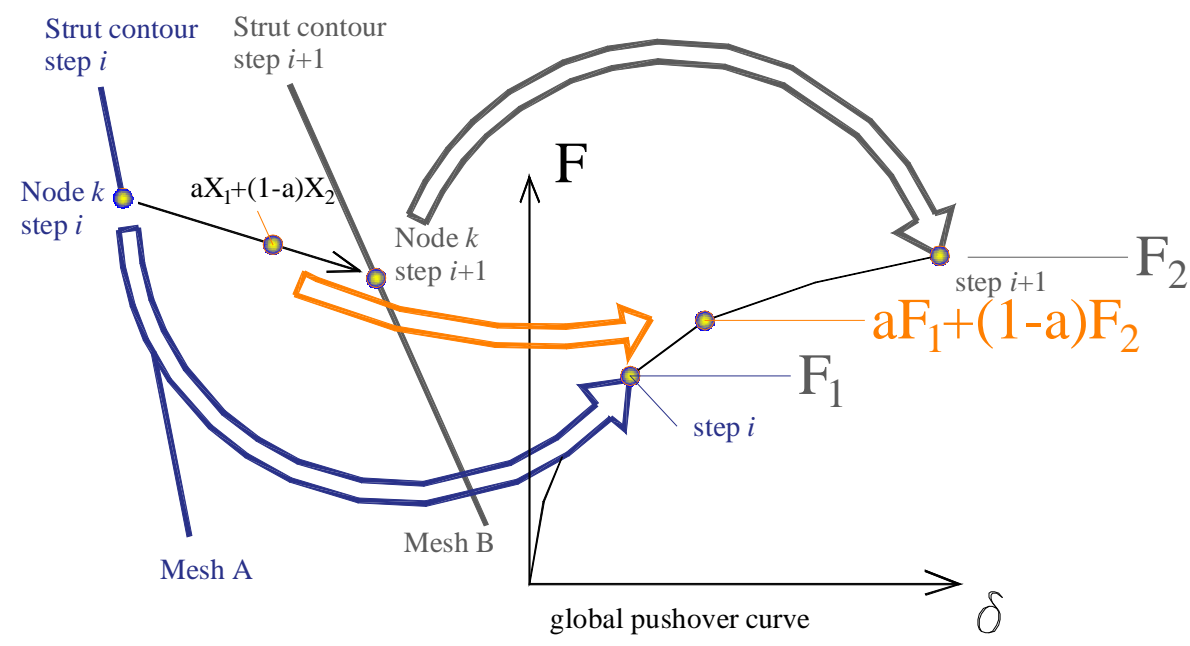

Figure 3: typical mesh adaptation at a structural level in order to smoothly follow the evolution of the compressed struts increasing the horizontal load.

\section{A NUMERICAL BENCHMARK INVESTIGATION}

In order to assess the novel two-step approach proposed, a three-storey masonry wall with three bays, see Figure 4, is analyzed in detail. The same benchmark has been already analyzed by Milani et al. (2009) with a variety of different approaches, including equivalent frames.

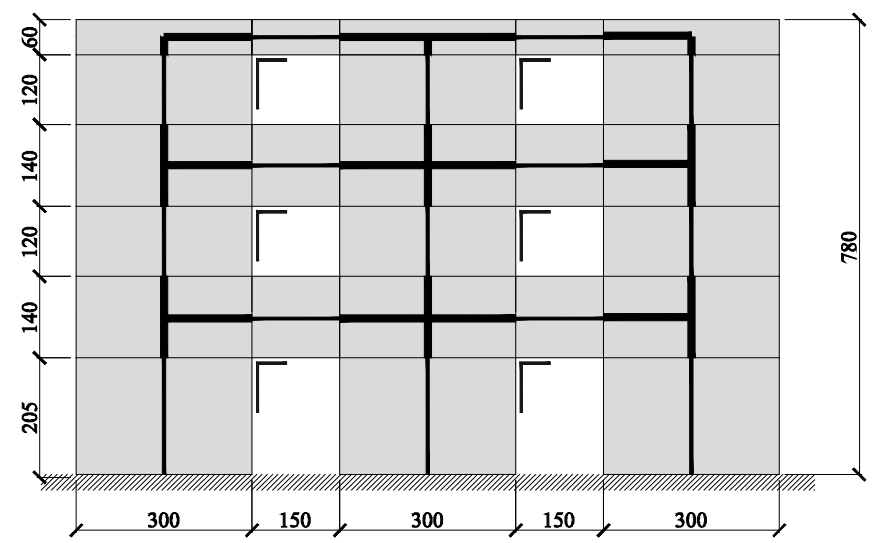

Figure 4: Geometry (all dimensions in $\mathrm{cm}$ ) and resulting equivalent frame model used in equivalent frame analyses for the considered masonry wall

Comparisons with respect to both standard equivalent frame pushover analyses and computationally expensive nonlinear 2D heterogeneous numerical models are therefore already at disposal.

The geometry of the structure is common for masonry buildings in the area of European Alps. All piers are $3 \mathrm{~m}$ long with $1.5 \mathrm{~m}$ in between. A clear storey height of $2.4 \mathrm{~m}$ is considered. At each floor level, vertical loads representing distributed dead and live loads are equal to $14.2 \mathrm{kN} / \mathrm{m}$. For simplicity sake the self-weight of the masonry is supposed to act at the storey levels; the masonry density is assumed equal to $\gamma=1080 \mathrm{~kg} / \mathrm{m}^{3}$, which is again typical for hollow blocks.

The wall was simulated in Milani et al. (2009) assuming at storey level a set of elastic beams with transversal 30x30 section to mimic a technical case-study that could represent ring beams or floors with tie rods embedded having axial strength equal to $35.7 \mathrm{kN}$. The interested reader is however referred to Milani el al. (2009) on technical details regarding this issue.

Four different types of models and the corresponding results are at disposal to benchmark the present two-step model. They are the following: 
(i) Frame models: two equivalent frame models are used to compute the pushover curve of the investigated wall. The first is a non standard software with a peculiar behavior of the spandrels originally proposed by Milani et al. (2009). It is characterized by a determination of the ultimate load carrying capacity (both for shear and bending moment) of the spandrels through a FE heterogeneous limit analysis procedure performed on isolated elements, see also Milani et al. (2006). The behavior of the piers is assumed according to Italian Code recommendations.

The second model is again an equivalent frame where ultimate strength of plastic hinges of both coupling beams and piers is determined according to the equations provided by the Italian Code. The capacity of a masonry spandrel with no tension member should not be considered in design, but if a tension member is introduced the ultimate bending moment and ultimate shear capacity of a single spandrel can be evaluated as follows:

$$
\begin{aligned}
& \left.M_{u}=H_{p} h / 2 \mid 1-H_{p} /\left(0.85 f_{h d} h t\right)\right\rfloor \\
& V_{u}=f_{v k o} h t
\end{aligned}
$$

where $t$ is the masonry thickness, $h$ is the spandrel height, $f_{h d}$ is the masonry design compressive strength for loads parallel to the bed joints, $f_{v k o}$ is the design shear resistance of masonry in the absence of compression. $H_{p}$ stands for the minimum value of $0.4 f_{h d} h t$ and of the axial strength of the tension element (tie-rod, ring beam, etc.) that withstand tensile stresses generated by the inclined compressive strut in the spandrel. Here the presence of tie rods at a floor level with pretension equal to $35.7 \mathrm{kN}$ is considered, so that spandrels can exhibit a certain ultimate carrying capacity, which is more realistic. The assumption is again made in agreement with the results presented by Milani et al. (2009).

(ii) Heterogeneous FE model: a computationally demanding heterogeneous FE 2D approach is also used to investigate the structure. Bricks are assumed elastic and modelled by means of quadrilateral elements. Mortar joints are reduced to interfaces with a Mohr-Coulomb failure surface. The geometry of the model is hence the same as for the limit analysis (Model (iii)). The analysis is a conventional non-linear approach. It provides a relatively detailed prediction of the actual behavior of the wall, but an extensive computational effort is required. The heterogeneous analyses where originally carried out in Atena software by Milani et al. (2009), but here are performed again using the non-commercial software proposed by Milani (2011).

(iii) Limit analysis: a heterogeneous limit analysis of the entire wall is finally carried out to predict the ultimate load bearing capacity and the collapse mechanism. The methodology is that presented for the first time in Milani et al. (2006) and used in Milani et al. (2009). Through limit analysis, only the ultimate capacity with the corresponding collapse mechanism of the system can be determined, meaning that a detailed pushover curve cannot be retrieved. An associated flow rule is assumed, meaning that frictional phenomena are accounted for in an approximate way. The benefit is that the estimation of the ultimate load bearing capacity occurs through linear programming, which proved excellent stability in most cases, along with reduced computational costs.

In the pushover analyses, the models are loaded by a lateral force that is triangularly distributed over the height of the wall. The corresponding point loads are concentrated at the storey levels. For the equivalent frame model, a reduced Young's modulus for masonry is adopted ( $E_{M}=2340 \mathrm{MPa}$ ) in order to account for: (a) masonry cracking at low levels of lateral load, and (b) deformability of the boundary zones (which cannot be modelled in an equivalent frame approach featuring rigid offsets).

The discretization used for the structural computations can be conveniently derived from the load path achieved through the topology optimization approach presented in Section 2.1. Figure 5 shows the optimal layouts (strut-only load paths) that are computed as a result of Eqn. (2), when enforcing the same volume fraction (35\%) and adopting a single load approach $(L=1)$. Each simulation includes the vertical forces and a different amount of horizontal forces, intended as a percentage of the vertical ones. 


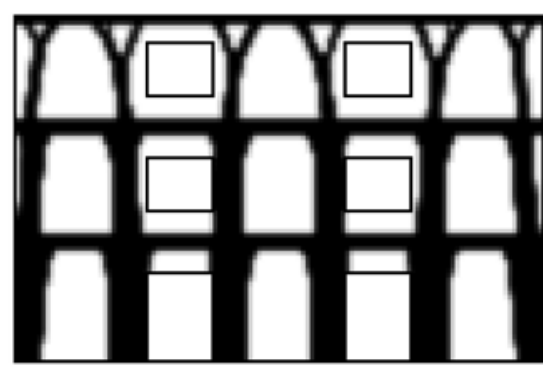

(a)
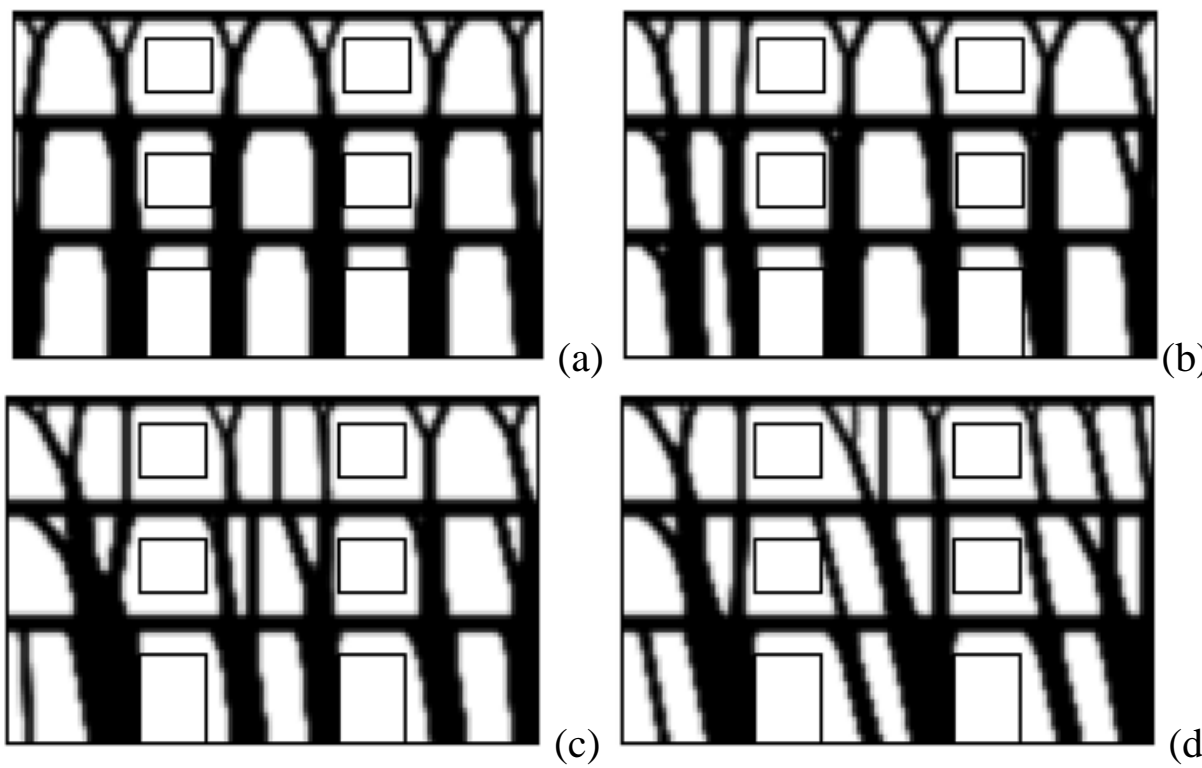

(b)
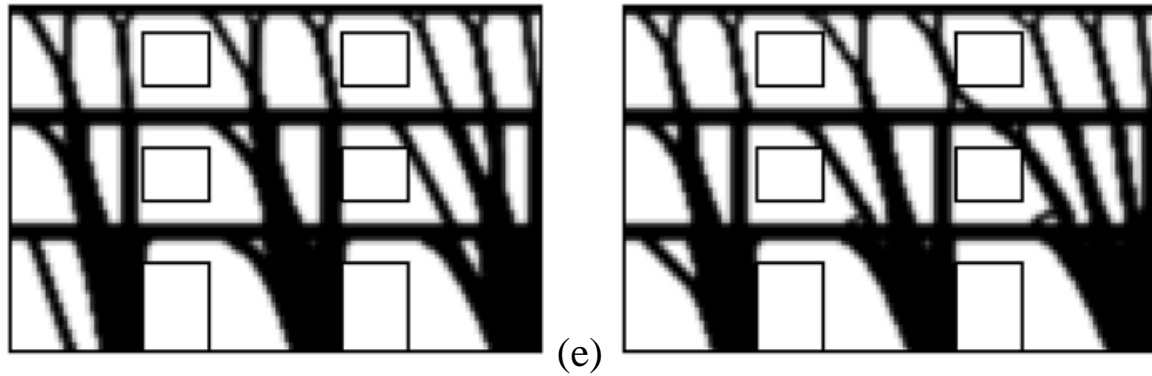

(d)
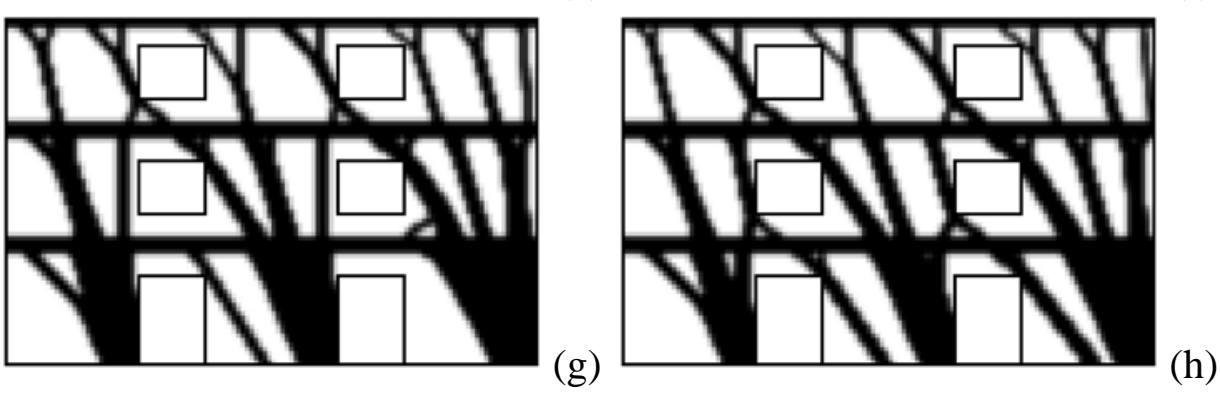

Figure 5: Result of the topology optimization, compression-only material model with vertical loads and horizontal loads equal to 0.00 (a), 0.05 (b), 0.10 (c), 0.15 (d), 0.20 (e), 0.25 (f), 0.30 (g), 0.35 (h) times the vertical ones. 
Mesh 1
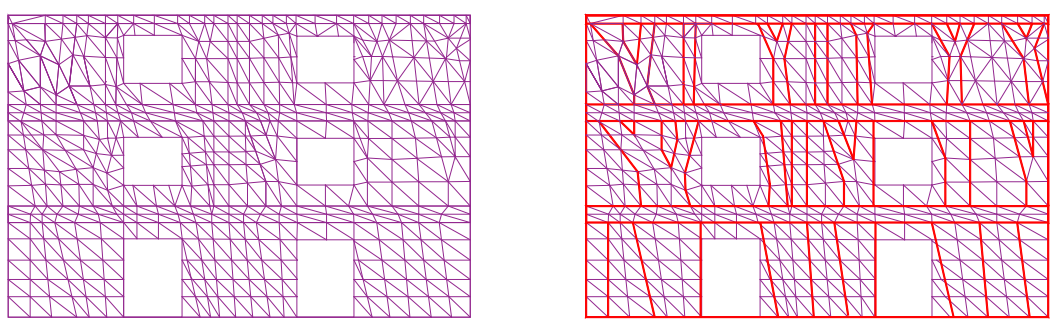

Mesh 2
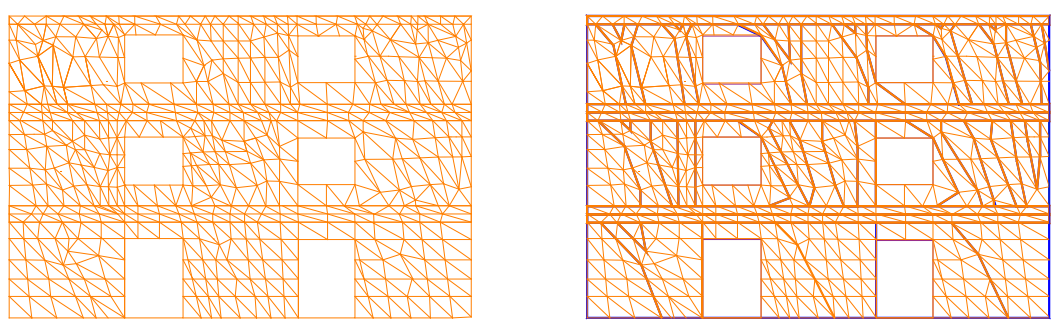

Mesh 3

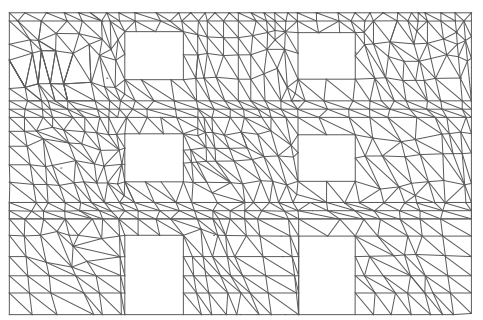

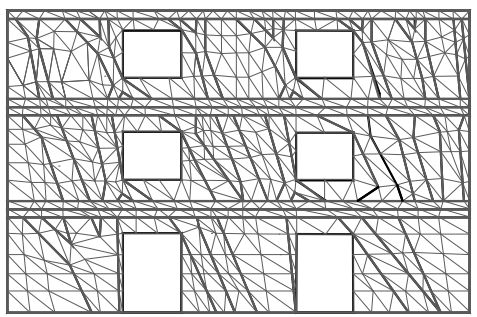
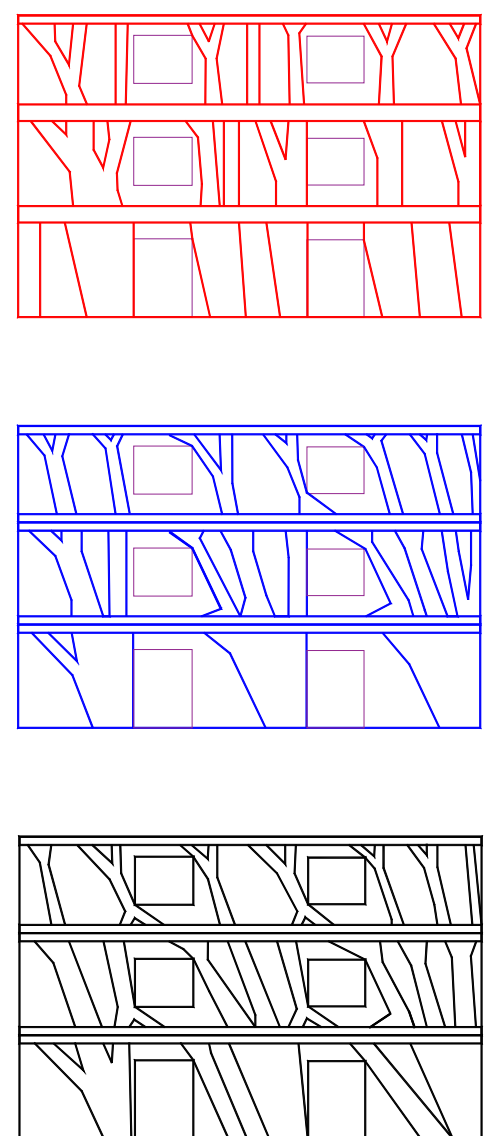

Figure 6: Three FE discretizations used to perform the evolutionary mesh-pushover analyses. Mesh 1 is used from $\mathrm{r}=0$ to 0.10. A combination of Mesh 1 and 2 from 0.10 to 0.25 . A combination of Mesh 2 and 3 from 0.25 to the end of the simulations (r ratio between horizontal and vertical loads)

In all the pictures black regions stand for material regions, whereas white ones mean void zones. As one may easily see, the achieved optimal load path (black regions) depends on the amount of lateral loads. This would suggest the adoption of an adaptive strategy to derive an appropriate mesh for each one of the load steps considered in the pushover analysis. To speed up the computation, Eqn. (2) can be conveniently implemented in its general form for multiple loads, considering $L=8$ in order to include the different load conditions that were separately considered in Figure 5 . This is to achieve a strut-only layout that minimizes the sum of the relevant strain energies, with the aim of finding a load path that is appropriate for each one of the considered load steps. The achieved optimal topology is depicted in Figure 2.

Standard post-processing techniques allow deriving contour plots of the optimal struts, whose axes can be used to generate the triangular mesh for the pushover analysis. Figure 6 shows the optimal discretizations achieved processing some of the optimal layouts found in Figure 5: Mesh 1 is obtained with a horizontal load equal to 0.1 of the gravity loads, Mesh 2 with 0.25 and Mesh 3 with 0.35. Assuming as $r$ the ratio between horizontal and vertical loads, Mesh 1 is therefore used in the structural analyses for $r$ ranging between 0 and 0.1, a combination of Mesh 1 and 2 for $r$ between 0.10 to 0.25 and a combination of Mesh 2 and 3 for $r$ between 0.25 and the end of the simulations. 
Table 1. Properties assumed for the interfaces allowing for failure along mortar joints and within bricks (a) and elastic properties assumed for the masonry (b-c). $f_{t}$ Tensile strength, $f_{c}$ Compressive strength, $\Phi$ Friction angle, $\Phi_{2}$ Shape of the linearized compressive capacity, c Cohesion

\begin{tabular}{|c|c|c|c|c|c|}
\hline & $f_{t}[\mathrm{MPa}]$ & $f_{c}[\mathrm{MPa}]$ & $\Phi$ & $\Phi_{2}$ & $c[\mathrm{MPa}]$ \\
\hline Mortar reduced to interfaces & 0.06 & 8 & $37^{\circ}$ & $80^{\circ}$ & $1.4 f_{t}$ \\
\hline Brick-brick interfaces & & & $45^{\circ}$ & & 2.0 \\
\hline Masonry elastic modulus & $E_{m}[\mathrm{MPa}]$ & 5770 & \multirow{2}{*}{\multicolumn{3}{|c|}{ (*) Note: homogenized values }} \\
\hline Masonry Shear modulus & $G_{m}[\mathrm{MPa}]$ & 2140 & & & \\
\hline Mode 1 mortar fracture energy & GI [N/mm] & 0.0005 & & & \\
\hline Mode 2 mortar fracture energy & GII [N/mm] & 0.0004 & & & \\
\hline
\end{tabular}

Mechanical properties used in the computations of the two-step model are reported in Table 1. A homogenization approach already presented in Milani (2011) is used to evaluate stress-strain behavior of the interfaces at a structural level at different orientation of the interface with respect to the horizontal direction. Such stress-strain curves (horizontal and vertical direction and shear) and the linear piecewise constant approximations used for the computations are represented in Figure 7. 


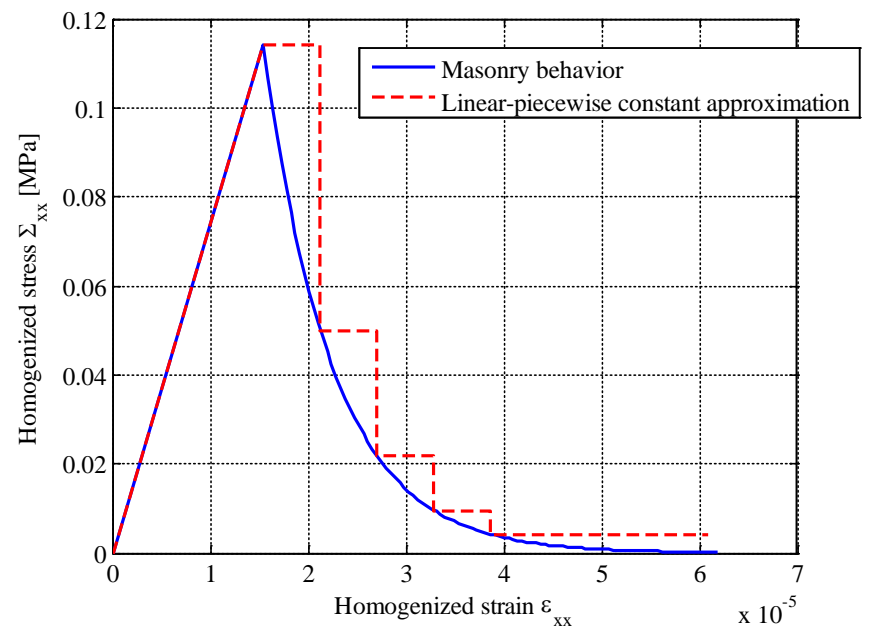

(a)

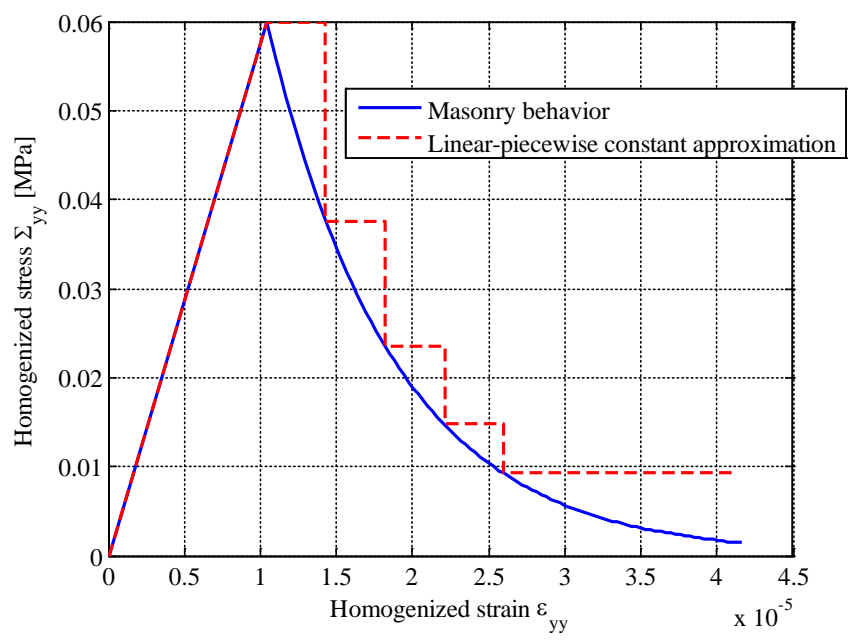

(b)

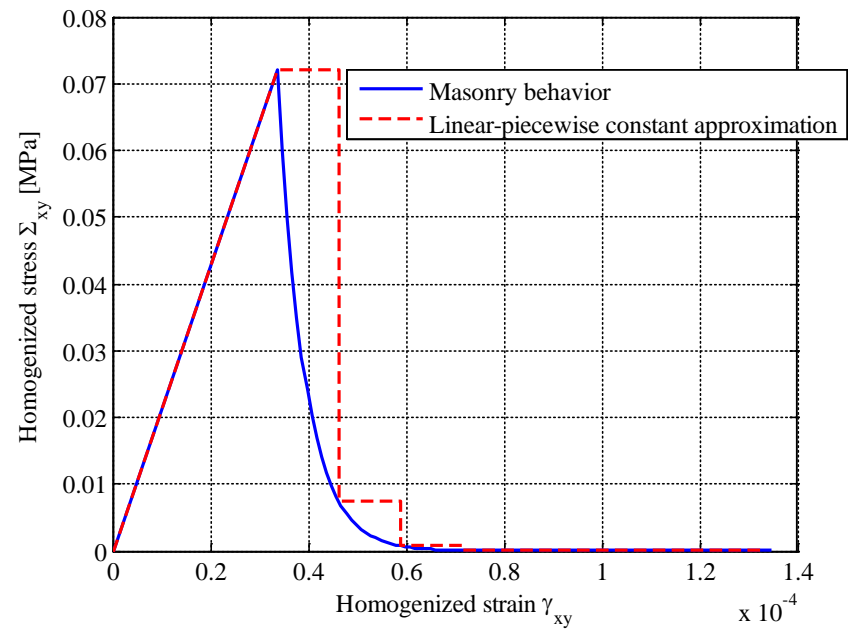

(c)

Figure 7: Homogenized stress-strain behaviour for the structural level and linear-piecewise constant approximation used in the FE computations.

The global base shear-displacement of the control node (assumed in the middle of the last storey level) obtained with the previously presented approaches are provided in Figure 8. Five curves are represented, corresponding to pushover global behaviors provided by frame models (i) (two 
curves), full 2D heterogeneous approach (model (ii), one curve) and present procedure (two curves), plus a collapse load provided by limit analysis model (iii).

The present approach has been applied both by means of the adaptive scheme with meshes reported in Figure 6 and with the coarse FE discretization achieved from the optimal solution for multiple loads shown in Figure 2.

As can be pointed out, the agreement between the heterogeneous FE model, which reasonably represents the reference solution, and the present approach is strong. It is more satisfactory than those obtained with equivalent frame models, meaning that a comparable computational cost provides results that are more close to a realistic behavior. Only the coarse mesh (which is not adaptive), results stiffer in the initial elastic range, a well-known drawback of coarse meshes with rigid elements and tends to concentrate softening in an unrealistic manner, again due to the insufficient number of interfaces used. In any case the outcome seems better than those provided by the equivalent frames.

Deformed modes at collapse are depicted in Figure 9 for the sake of completeness, as obtained with the aforementioned different numerical models.

In addition, it is interesting to highlight that capacities obtained with the frame models exhibit always a stiffer behavior, also because of the present of fictitious rigid links on cross areas, which obviously do not exist in 2D models.

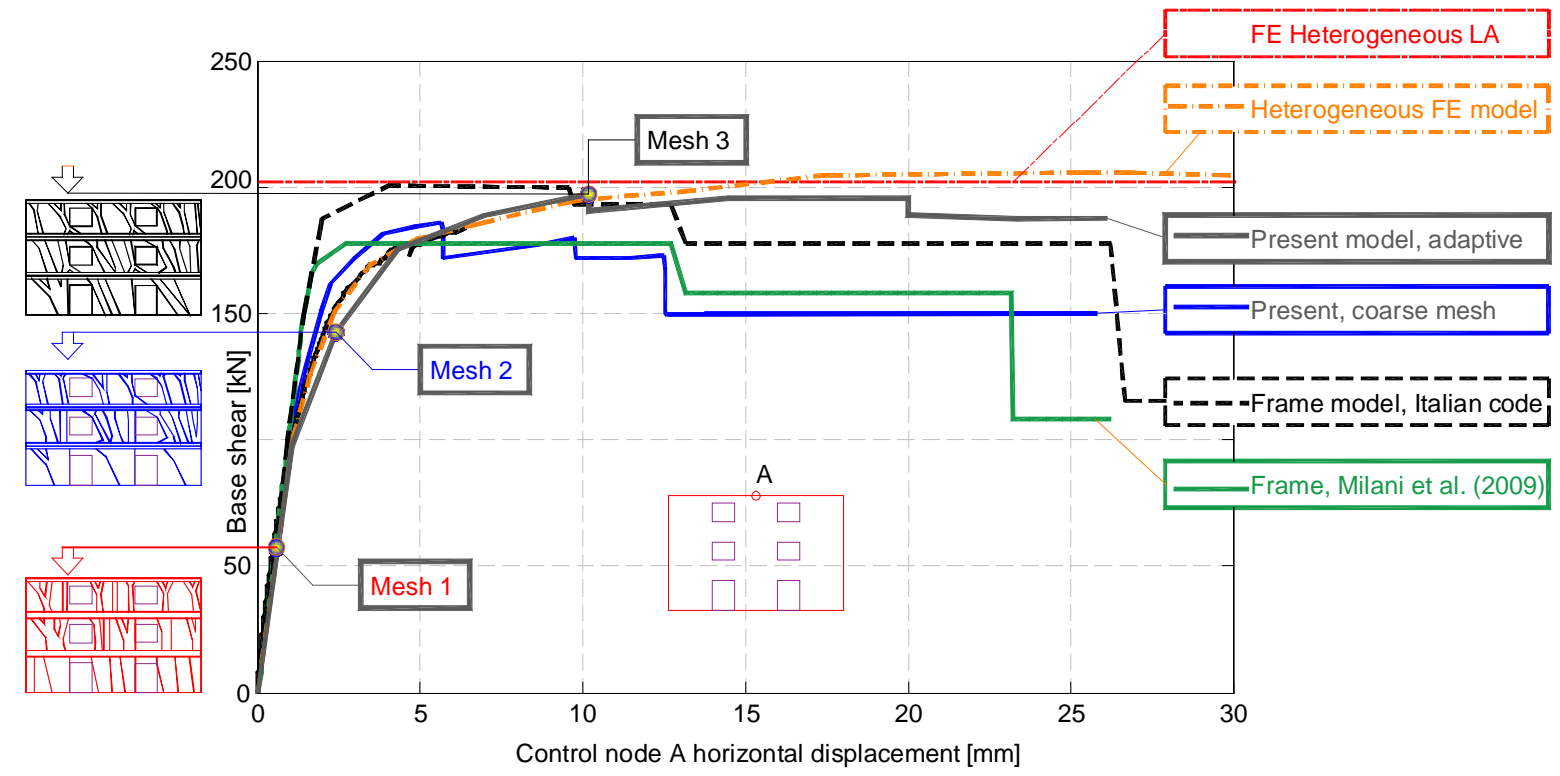

Figure 8: Pushover curves for the different models considered in this paper.

From an analysis of deformed shapes at collapse obtained with the different methods, it is interesting to point out that all of them suggest the arising of failure mechanisms that are characterized by bending failure of the three base piers and shear failure of all spandrels (see indication of the plastic hinges reported in the equivalent frame model in Figure 9). Only the coarse mesh of subfigure (e) seems not perfectly able to develop in the inelastic phase a rocking of the ground floor left pier, probably because of the insufficient refinement of the mesh used. It is therefore recommended for practical purposes, whilst the global pushover curves is still realistic in this benchmark, to utilize medium refinements of the discretizations adopted. As expected, the heterogeneous finite element analysis provides further insight on the arising failure mechanisms, see e.g. the inclined fractures from the top left corner of the doors to the bottom floor in subfigure (d), but paying a much higher computational cost. 


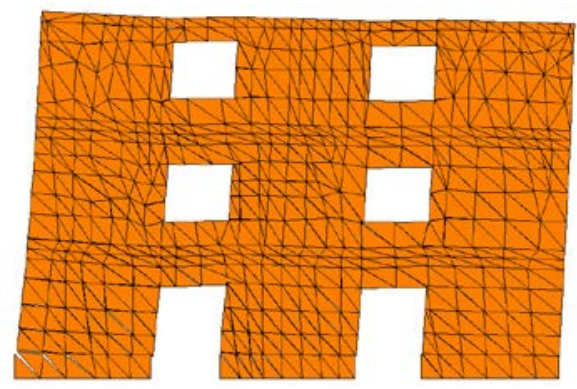

(a)

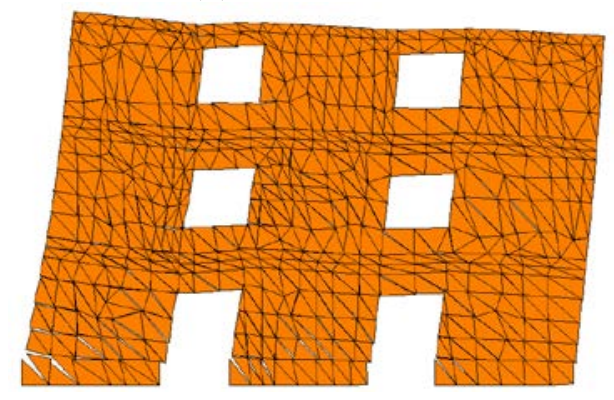

(b)

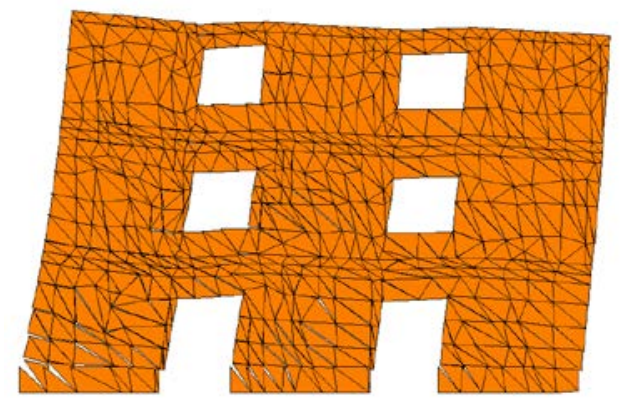

(c)

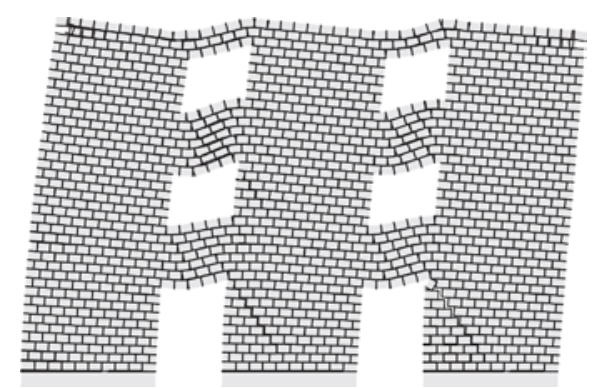

(d)

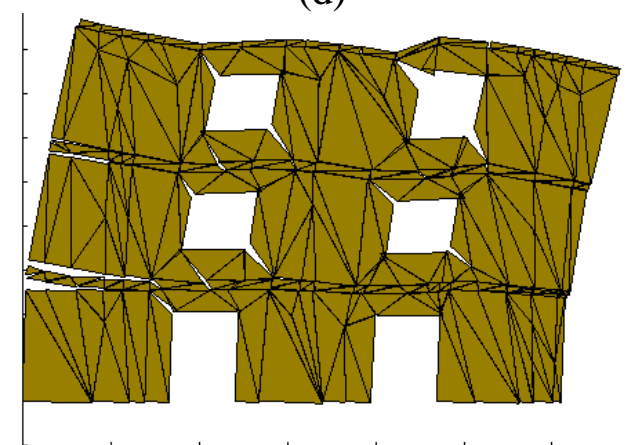

(e)

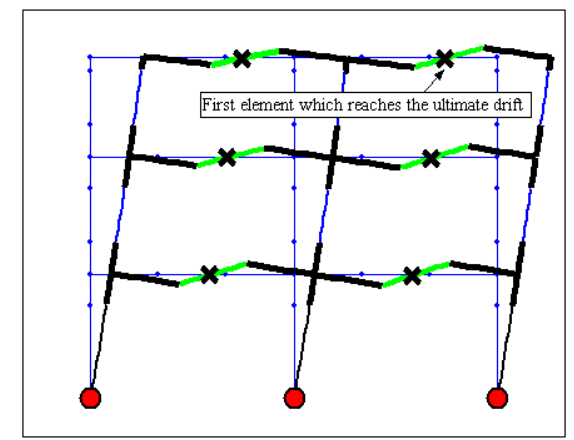

(f)

Figure 9: Deformed shape at collapse via different approaches. (a)-(c): adaptive present two-step method, Mesh 1-3. (d): heterogeneous FE model. (e) not adaptive present two-step method, coarse mesh. (f) equivalent frame model (red circles: in-plane bending moment failure, black crosses: shear failure).

\section{CONCLUSIONS AND PERSPECTIVES}

A two-step approach has been discussed to address the pushover analysis of in-plane loaded masonry walls. A suitable problem of topology optimization for compression-only truss-like structures has been formulated to find a no-tension load path for the masonry structure acted upon by seismic forces. Different values of the horizontal action have been considered to derive an adaptive mesh or an optimal discretization that is suitable for multiple loads. Rigid triangular elements have been used whose interfaces are assumed to behave as elasto-plastic with softening in both tension and compression (with peak tensile strength almost vanishing). Finally, the pushover analysis has been performed on the improved discretizations.

The two-step procedure does not call for any a-priori assumption on the location of the triangular elements and on the length of the rigid offsets. No rigid connection is required by the proposed method.

A preliminary investigation has been introduced in this work on a well-known benchmark with low complexity, showing good performance of the proposed algorithm. 
The proposed approach is ideally suited to cope with structures of any shape and geometry. The topology optimization approach can be easily applied to more complex buildings, thus allowing for the adoption of accurate pushover analysis on historic constructions.

Indeed, simulations regarding panels framed in the three-dimensional space will be addressed in the next future. Also, the adoption of the adaptive strategy will be modified in order to make it fully independent from user's choices.

\section{REFERENCES}

Addessi, D., Libertatore, D., Masiani, R. 2015. Force-based beam finite element (FE) for the pushover analysis of masonry buildings. International Journal of Architectural Heritage 9(3): 231-243.

Belmouden, Y., Lestuzzi, P. 2009. An equivalent frame model for seismic analysis of masonry and reinforced concrete buildings. Construction and building materials; 23(1): 40-53.

Bendsøe, M.P. 1989. Optimal shape design as a material distribution problem. Structural Optimization; 1: 193-202.

Bendsøe, M.P., Sigmund, O. 2003 Topology optimization theory, methods and applications. New York. Springer.

Bruggi, M. 2010. On the automatic generation of strut and tie patterns under multiple load cases with application to the aseismic design of concrete structures. Advances in Structural Engineering; 13(6): 1167-1181.

Bruggi, M. 2014. Finite element analysis of no-tension structures as a topology optimization problem. Structural and Multidisciplinary Optimization; 50(6): 957-973.

Bruggi, M. 2016. A numerical method to generate optimal load paths in plain and reinforced concrete structures. Computers \& Structures; 170: 26-36.

Bruggi, M., Duysinx, P. 2013. A stress-based approach to the optimal design of structures with unilateral behavior of material or supports. Structural and Multidisciplinary Optimization; 48(2): 311326.

Cai, K. 2011. A simple approach to find optimal topology of a continuum with tension-only or compression-only material. Structural and Multidisciplinary Optimization; 43: 827-835.

Chen, S.Y., Moon, F.L., Yi, T. A. 2008. A macroelement for the nonlinear analysis of in-plane unreinforced masonry piers. Engineering Structures 30(8): 2242-2252.

Clementi, F., Gazzani, V., Poiani, M., Lenci S. 2016. Assessment of seismic behavior of heritage masonry buildings using numerical modelling. Journal of Building Engineering 8: 29-47.

Duysinx, P., Bendsøe, M. 1998. Topology optimization of continuum structures with local stress constraints. International Journal for Numerical Methods in Engineering; 43:1453-1478.

Galasco, A., Lagomarsino, S., Penna, A., Resemini, S. 2004. Non-linear seismic analysis of masonry structures. Proc. of the 13th Word Conference on Earthquake Engineering (WCEE), Vancouver Canada.

Kwak, H.G., Noh, S.H. 2006. Determination of strut-and-tie models using evolutionary structural optimization. Engineering Structures; 28(10): 1440-1449. 
Lagomarsino, S., Penna, A., Galasco, A., Cattari, S. 2013. TREMURI program: an equivalent frame modelfor the nonlinear seismic analysis of masonry buildings, Eng.Struct. 56: 1787-1799.

Liang, Q.Q., Xie, Y.M., Steven, G.P. 2000. Topology optimization of strut-and-tie models in reinforced concrete structures using an evolutionary procedure. ACI Structural Journal; 97(2): 322-330.

Magenes, G., Della Fontana, A. 1998. Simplified non-linear seismic analysis of masonry buildings. Proc. of the British Masonry Society 1998; 8: 190-195.

Marquez, R., Lourenço, P.B. 2011. Possibilities and comparison of structural component models for the seismic assessment of modern unreinforced masonry buildings. Computers and Structures 89: 2079-2091.

Milani, G, Tralli, A. 2012. A simple meso-macro model based on SQP for the non-linear analysis of masonry double curvature structures. International Journal of Solids and Structures; 49(5): 808-834.

Milani, G. 2011. Simple homogenization model for the non-linear analysis of in-plane loaded masonry walls. Computers \& Structures; 89: 1586-1601.

Milani, G., Beyer, K., Dazio, A. 2009. Upper bound limit analysis of meso-mechanical spandrel models for the pushover analysis of 2D masonry frames. Engineering Structures; 31(11): 26962710 .

Milani, G., Lourenço, P.B., Tralli, A. 2006. Homogenised limit analysis of masonry walls - Part II: structural examples. Computers and Structures; 3-4: 181-195.

Milani, G., Tralli, A. 2011. Simple SQP approach for out-of-plane loaded homogenized brickwork panels accounting for softening. Computers \& Structures; 89(1-2): 201-215.

Pasticier, L., Amadio, C., Fragiacomo, M. 2008. Non-linear seismic analysis and vulnerability evaluation of a masonry building by means of the SAP2000 V.10 code. Earthquake Engineering and Structural Dynamics; 37: 467-485.

Rinaldin, G., Amadio, C., Macorini, L. 2016. A macro-model with nonlinear springs for seismic analysis of URM buildings. Earthquake Engineering and Structural Dynamics 45(14): 2261-2281.

Salonikios, T., Karakostas, C., Lekidis, V., Anthoine, A. 2003. Comparative inelastic pushover analysis of masonry frames. Engineering Structures; 25: 1515-1523.

Svanberg, K. 1989. Method of moving asymptotes-a new method for structural optimization. International Journal for Numerical Methods in Engineering; 24(3): 359-373.

Zhou, M., Rozvany, G.I.N. 1991. The COC algorithm, Part II: topological, geometrical and generalized shape optimization. Computer Methods in Applied Mechanics and Engineering; 89: 309-336. 